\title{
The Difference of Lymphocyte, hs-CRP, and Electroencephalogram with and without Simvastatin in Acute Ischemic Stroke
}

\author{
Chairil Amin Batubara ${ }^{1}$, Aldy Safruddin Rambe ${ }^{1}$, Nindia Sugih Arto $^{2}$ \\ ${ }^{1}$ Department of Neurology, Faculty of Medicine, Universitas Sumatera Utara/Adam Malik Hospital, Medan, Indonesia. \\ E-mail:cabtbr@gmail.com; chairil-amin@usu.ac.id. \\ ${ }^{2}$ Department of Clinical Pathology, Faculty of Medicine, Universitas Sumatera Utara/Adam Malik Hospital, Medan, Indonesia
}

\begin{abstract}
Mortality and morbidity due to stroke rank the highest in Indonesia (15.4\%), and most types of stroke are ischemic (87\%). Inflammation has a role in the pathophysiology of both ischemic stroke and also provokes acute symptomatic epileptic seizures (3-6\%) in the first 7 days after stroke. Statins have been used for the treatment of dyslipidemia in stroke patients. Some studies showed that statins reduced the inflammatory response after a stroke and prevented the recovery of epileptic seizures. This study aimed to determine the differences in lymphocytes, hs-CRP, Electroencephalogram (EEG) with and without Simvastatin in acute ischemic stroke. This research was an experimental study with a double-blind, randomized control trial design consisting of two groups, a group given Simvastatin $20 \mathrm{mg} /$ day, and a group given a placebo for seven days. The difference in lymphocytes, hs-CRP, EEG, and epileptic seizures between the two groups were then analyzed. The sample was 26 people, consisting of 17 (65.4\%) males and 9 (34.6\%) females with an average age of $59 \pm 5.8$ years. Chi-Square and Fisher's test showed a significant difference in hs-CRP $(p=0.005)$ and epileptic seizures $(p=0.015)$, but no significant difference in lymphocytes $(p=0.336)$ and EEG $(p=0.42)$ between groups given Simvastatin $20 \mathrm{mg} /$ day and those given placebo. There was a significant difference in hs-CRP and epileptic seizures, but no significant difference in lymphocyte count and EEG between the two groups with and without Simvastatin administration.
\end{abstract}

Keywords: Lymphocytes, hs-CRP, EEG, epileptic seizure, acute ischemic stroke

\section{INTRODUCTION}

Mortality and morbidity due to stroke in Indonesia rank the highest (15.4\%), and the stroke prevalence has increased from $8.3 \%$ in 2007 to $12.1 \%$ in 2013. ${ }^{1}$ The most common types of stroke are ischemic (87\%), intracerebral hemorrhage (10\%), and subarachnoid bleeding (3\%). ${ }^{2,3}$

Stroke can be followed by acute symptomatic epileptic seizures (3-6\%) within the first 7 days and epilepsy (12\%) after the first 7 days known as epileptic seizures without provocation at least once. The incidence of acute symptomatic epileptic seizures is higher in intracranial hemorrhagic (10-16\%) than ischemic stroke (2-4\%). Symptomatic acute epileptic seizures and epileptic seizures without provocation correlates with poor functional outcome, and increased mortality. ${ }^{4}$ Risk factors of epileptic seizures include cortex lesion, a site at anterior hemisphere, and cardioembolic stroke. ${ }^{5}$

The pathophysiology of acute ischemic stroke is an inflammatory process, which involves endothelial activation, blood-brain barrier disruption, accumulation of inflammatory mediators, and infiltration of leukocytes and platelets. ${ }^{6}$ There have been much numerous clinical evidence and experimental studies which showed that epileptic activity is related to the inflammatory process. ${ }^{7}$

Early EEG abnormalities are independent predictors of functional stroke outcomes. Poor outcomes are associated with slow $(p<0.001$ ) and asymmetric $(p<0.001)$ activity of Electroencephalogram (EEG) background. Death in the first year after stroke was associated with acute symptomatic epileptic seizure $(p=0.015)$ and suppression of EEG $(p=0.019){ }^{8}$

Early increase of neutrophils but no increase of lymphocytes is associated with the increase in stroke volume. ${ }^{9}$ Cerebral ischemia can trigger some acute responses, which are monitored by a significant increase in high sensitivity CRP levels (hs-CRP) and acute ischemic stroke, especially during the first few weeks. The hs-CRP level is a strong risk factor for death in acute ischemic stroke. The level of hs-CRP is increased in the case of stroke, which shows an inflammatory response in acute stroke. ${ }^{10}$ The increased hs-CRP levels in patients with generalized motor epileptic seizures, which occur every day, are 
more frequent than other types of seizures and controls. A significant association was found in CRP levels with tonic-clonic epileptic seizures. ${ }^{11}$

The administration of anticonvulsants as prophylaxis for epileptic seizures in stroke remains poorly recommended by the American Heart/Stroke Association (AHA/ASA) and European Stroke Organization (ESO). ${ }^{4.12}$ Observational studies have found that the use of statins in the acute phase of ischemic stroke can reduce the risk of acute symptomatic epileptic seizures ( $p<0.001)$, despite no significant relationship to post-stroke epilepsy $(p=0.349){ }^{13}$ It has been proven that statins inhibit recruitment, adhesion, and migration of inflammatory cells. Statins are also associated with a decrease in inflammatory biomarkers such as CRP and cytokines (interleukins 1, 6 and 12 ). ${ }^{14}$

\section{METHODS}

This research was an experimental study with a double-blind, randomized control trial design consisting of 2 groups, such as a group given Simvastatin $20 \mathrm{mg} / \mathrm{day}$, and a group was given a placebo for seven days. The study was performed at the Department of Neurology and the Department of Clinical Pathology of the General Hospital Adam Malik Medan, from August to November 2019. A large sample of 26 subjects was randomly obtained and double-blinded, divided into 16 subjects receiving Simvastatin $20 \mathrm{mg} /$ day and the other 16 receiving placebo as controls. Inclusion criteria were: patients with acute ischemic stroke (onset $\leq 7$ days) and gave consent to participate in this study. Exclusion criteria were: subjects with recurrent ischemic stroke, patients with severe comorbidities (acute coronary syndromes, neoplasms, autoimmune diseases, electrolyte disturbances and severe blood sugar levels that could not be corrected), patients with inflammation and infection, any history of immunosuppressant drugs and statins use, hematological disorders and impaired liver and kidney function. Ethical Clearance was obtained from the Health Research Ethics Commission of the Faculty of Medicine, Universitas Sumatera Utara (FK USU)/Adam Malik Hospital, Medan with a number of 679/TGL/ KEPK FK USU-RSUP HAM/2019.

Acute ischemic stroke patients (onset $\leq 7$ days) who met the inclusion and non-exclusion criteria were randomly divided into 2 groups and double-blinded into groups given Simvastatin 20 mg and groups given a placebo for seven days (acute phase). The epileptic seizure was observed in both groups. On the $7^{\text {th }}$ day, a complete blood count, hs-CRP levels measurement, and an EEG test were performed to determine the differences between the two groups. The parameters were as follows: Lymphocyte count. Normal: $25-40 \%$, decreased: $<25 \%$ and normal-increase: $\geq 25 \%$; hs-CRP levels, increases: $>3 \mathrm{mg} / \mathrm{dL}$ and no increase: $\leq 3 \mathrm{mg} / \mathrm{dL}$; EEG overview. Mild to moderate abnormality: base rhythm $<8 \mathrm{~Hz}$, overall intermittent deceleration or one region/hemisphere, excessive beta, and asymmetry. Severe abnormal: epileptiform, EEG seizures, and regional or overall continuous slowdown; The presence or absence of epileptic seizures. ${ }^{15}$

Data were analyzed using the Chi-Square or Fisher test (if it does not meet the Chi-Square test requirements). Statistical tests were considered significant if the $p$-value $<0.05$.

The subject's blood was withdrawn through the venipuncture from the median cubital vein. The vein was first cleaned with $70 \%$ alcohol and allowed to dry. Blood was drawn using $3 \mathrm{ml}$ of venoject in the K2EDTA vacutainer tube and $5 \mathrm{~mL}$ in the vacutainer gel clot activator tube $(3 \mathrm{~mL})$. The K2EDTA vacutainer tube was slowly homogenized eight times. This test must be completed within one hour after sampling. The blood in the vacutainer gel clot activator tube was allowed to freeze for 20 minutes at room temperature, centrifuged at $3,000 \mathrm{rpm}$ for 20 minutes, and the serum was separated and put in a 1 $\mathrm{mL}$ plastic tube (aliquot). The serum for measurement of a hs-CRP level was stored in the freezer $-20^{\circ} \mathrm{C}$ until the specified examination time (maximum six months). ${ }^{16}$ Lymphocyte count was determined using automatic cell counting Sysmex XT-4000i through a Complete Blood Count (CBC) test based on the method of flow cytometry. Measurement of hs-CRP levels was carried out using Cobas C 501 analyzer after an adequate number of samples have been collected. The frozen material was thawed at room temperature and homogenized with a vortex. The control solution was also adjusted with room temperature $\left(20-25^{\circ} \mathrm{C}\right)$. The test was performed based on the principle of the particle-enhanced immunoturbidimetric assay. The R1 (buffer) reagents were firstly added to the material, then R1 (buffer) reagents added R2 (latex Anti CRP antibodies) were added respectively. Anti CRP antibodies bound to the latex microparticle will react with antigens in the sample to form $A g-A b$ complexes. The agglutination of the Ag-Ab complex is measured in a turbidimetric manner. ${ }^{10,17}$ 


\section{RESULTS AND DISCUSSIONS}

The study found 26 subjects with acute ischemic stroke, consisting of 17 males (65.4\%) and 9 (34.6\%) females. All participants had a mean age of $59 \pm 5.8$ years, with an age range of 47 years to 72 years with the highest number (15 subjects; $57.7 \%$ ) found in the age group of 57-66 years. Meanwhile, the least number (2 subjects; $7.7 \%$ ) was found in the age group of 67-76 years.

Fisher's exact test showed no significant differences in lymphocyte count between-group given Simvastatin 20 mg/day and groups with placebo $(p=0.336)$ (Table 1).

Chi-Square test showed significant differences in hs-CRP levels between the group given Simvastatin $20 \mathrm{mg} /$ day and group with placebo $(p=0.005)$ (Table 2).

Chi-Square test showed no significant difference in the Electroencephalogram (EEG) results between groups given Simvastatin $20 \mathrm{mg} /$ day and group given placebo $(p=0.42)$ (Table 3$)$.

Fisher's exact test showed a significant difference in the presence or absence of epileptic seizures between-group given Simvastatin $20 \mathrm{mg} /$ day and group were given a placebo $(p=0.015)$ (Table 4).

Table 1. Differences of lymphocyte count between simvastatin and placebo group in patients with acute ischemic stroke

\begin{tabular}{llcccc}
\hline & & $\begin{array}{c}\text { Simvastatin } \\
\mathbf{2 0} \mathbf{~ m g / d a y}\end{array}$ & Placebo & Total & \multirow{2}{*}{$\mathbf{p}^{*}$} \\
\hline \multirow{2}{*}{ Lymphocyte count } & Decreased $(<25 \%)$ & 5 & 3 & 8 & \multirow{2}{*}{0.336} \\
Total & Normal-increased $(\geq 25 \%)$ & 8 & 10 & 18 & \\
\hline
\end{tabular}

*Fisher's test

Table 2. Differences of hs-CRP levels between simvastatin and placebo group in patients with acute ischemic stroke

\begin{tabular}{llcccc}
\hline & & $\begin{array}{c}\text { Simvastatin } \\
\mathbf{m g} / \text { day }\end{array}$ & Placebo & Total & $\mathbf{p}^{*}$ \\
\hline \multirow{2}{*}{ hs-CRP levels } & Increased $(>3 \mathrm{mg} / \mathrm{L})$ & 2 & 9 & 11 & 0.005 \\
Total & Not increased $(\leq 3 \mathrm{mg} / \mathrm{L})$ & 11 & 4 & 15 & \\
\hline
\end{tabular}

*Chi-Square test

Table 3. Differences of EEG results between simvastatin and placebo group in patients with acute ischemic stroke

\begin{tabular}{llcccc}
\hline & & $\begin{array}{c}\text { Simvastatin 20 } \\
\mathbf{m g} / \text { day }\end{array}$ & Placebo & Total & $\mathbf{p}^{*}$ \\
\hline \multirow{2}{*}{ EEG results } & Severe abnormal & 7 & 9 & 16 & 0.42 \\
Total & Mild-moderate abnormal & 6 & 4 & 10 & \\
\hline
\end{tabular}

* Chi-Square test

Table 4. Differences in presence and absence of epileptic seizures between Simvastatin and placebo group in patients with acute ischemic stroke

\begin{tabular}{lccccc}
\hline & & $\begin{array}{c}\text { Simvastatin } \\
\mathbf{2 0} \mathbf{~ m g / d a y}\end{array}$ & Placebo & Total & \multirow{2}{*}{$\mathbf{p}^{*}$} \\
\hline Epileptic seizures & Present & 1 & 7 & 8 & \multirow{2}{*}{0.015} \\
Total & Absent & 12 & 6 & 18 & \\
\hline
\end{tabular}

*Fisher test 
In this study, there was a relatively higher decrease of lymphocytes counts in the Simvastatin group compared to the placebo group. However, the difference was not significant $(p=0.336)$. A study by Hillyard et al. found the same results; however, the Simvastatin was given to healthy subjects, and subjects with cardiovascular disease and insignificant results of decreased lymphocyte proliferation were found $(p=0.06)$. The mechanism by which statins can reduce lymphocyte values is not fully understood, presumably through disruption of the lymphocyte membrane lining. ${ }^{18}$ Lymphocytes play an important role in repairing inflamed tissues. Repair by lymphocytes is related to the release of cytokines and growth factors, which then activate microglial. Lymphocytes have a negative relationship with mortality in ischemic stroke. ${ }^{6}$ In this study, lymphocyte decrease was not significantly different between the two groups; therefore, statin administration would not lead to a detrimental effect for acute ischemic stroke.

There were significant differences in hs-CRP levels between the Simvastatin and a placebo group $(p=0.005)$. Similar results were found in the study by Kitagawa et al., which found a significant decrease in hs-CRP levels after statin (pravastatin) administration in ischemic stroke patients compared to controls $(p=0.007)$. An acute stroke can trigger an inflammatory response, which increases the CRP level. ${ }^{19}$ CRP is a glycoprotein produced by the liver and normally is not present in the blood. The presence of acute inflammation with tissue damage will stimulate CRP production. The increase of hs-CRP levels occurs 12-24 hours after stroke onset. Research shows that CRP plays a role in secondary brain damage after brain ischemia, possibly through complement activation, thereby aggravating tissue damage. $^{10}$ Statins can inhibit the recruitment, adhesion, and migration of inflammatory cells, which is characterized by a decrease in inflammatory biomarkers such as hs-CRP and cytokines. ${ }^{14}$

There were no significant differences in the EEG results $(p=0.42)$ between the two groups. Abnormal results were found in all subjects, with a severe abnormality in 16 subjects (61.5\%) and mild-moderate abnormality in 10 people (38.5\%). This was consistent with the research by Bentes et al., which found the abnormal slow activity of EEG results. Statins administration for seven days did not change the abnormality of EEG waves, presumably because Simvastatin was only given in a short time (acute phase). Research by Citraro et al., which conducted experiments on animals suggested that statins would have an effect on brain waves, after one month of use. ${ }^{20}$ This was also in accordance with AHA/ASA recommendations that statins are best given through the acute phase, which is up to 21 days after ischemic stroke onset. ${ }^{12}$

This study found a significant difference in epileptic seizures $(p=0.015)$ between the two groups. In the group given Simvastatin $20 \mathrm{mg} /$ day, less epileptic seizures were found (1 subject; $3.8 \%$ ) compared to the placebo group (7 subjects; $26.9 \%$ ). This finding was in accordance with the results of Guo et al., who found $3.4 \%$ of epileptic seizures which occurred at seven days of stroke onset (acute phase) and statin administration significantly reduced the risk of epileptic seizures in the acute phase $(p<0.001)$. Some proposed statin mechanisms to prevent seizures are: The acute ischemic stroke will increase extracellular glutamate, which can cause a burst of neurons. Statins can reduce the excitatory effects of glutamate by increasing glutamate uptake and modulating glutamate receptors (N-Methyl-D-Aspartate); Statins can reduce the volume of infarction through its anti-inflammatory, antioxidative, and inhibitory effects of neuronal apoptosis, wherein the volume of infarction is related to neuronal depolarization and epileptic seizures; Statins reduce the blood-brain barrier permeability and inflammation of the brain thereby preventing epileptic seizures. $^{13}$

\section{CONCLUSIONS AND SUGGESTIONS}

This study found significant differences in hs-CRP levels and epileptic seizures in the Simvastatin and placebo group, but no significant differences were found in lymphocyte counts and EEG results between the two groups.

Simvastatin can be given in the acute phase (the first 7 days of onset) ischemic stroke which can provide benefits to reduce inflammation in response to the acute phase, and can prevent epileptic seizures in the acute phase, to provide better outcomes for patients with acute ischemic stroke.

\section{REFERENCES}

1. Riskesdas (Riset Kesehatan Dasar). Jakarta, Badan Penelitian dan Pengembangan Kesehatan Kementerian Kesehatan Republik Indonesia, 2013; 123-126.

2. Go AS, Mozaffarian D, Roger V L, Benjamin E J, Berry J $D$, Borden W B, et al. Heart disease and stroke statistics 
2013 update: A report from the american heart association, Circulation, 2013; 127: e6-e245.

3. Batubara CA, Ritarwan K, Rambe AS. Effectiveness differences of Ranitidine and Omeprazole in prevention of stress ulcer and its effect on pneumonia occurrence and outcome of acute stroke patients IOP conference series: Earth and environmental science, 2018; 125: 012205.

4. Holtkamp $M$, Beghi $E$, Benninger $F$, Kalviainen $R$, Rocamora R, Christensen H. European Stroke Organisation guidelines for the management of post-stroke seizures and epilepsy. European Stroke Journal, 2017; 2(2): 103-115.

5. Kim BS, Sila C. Seizures in ischemic stroke. In: Koubeissi MZ, Alshekhlee A, Mehndiratta P (Eds.). Seizures in cerebrovascular disorders, a clinical guide. New York, Springer, 2015; 17-29.

6. Tokgoz S, Kayrak M, Akpinar Z, Seyithano A, Guney F, Betigul Yuruten B. Neutrophil lymphocyte ratio as a predictor of stroke. J Stroke Cerebrovasc Dis, 2013; 22(7): 1169-74.

7. Dambach $H$, Hinkerohe $D$, Prochnow $N$, Stienen MN, Moinfar Z, Haase CG, et al. Glia and epilepsy: Experimental investigation of antiepileptic drugs in an astroglia/microglia co-culture model of inflammation. Epilepsia, 2014; 55(1): 184-192.

8. Bentes C, Peralta AR, Martins H, Casimiro C, Morgado C, Franco AC, et al. Seizures, electroencephalographic abnormalities, and outcome of ischemic stroke patients. Epilepsia Open, 2017; 2(4): 441-452.

9. Segel GB, Halterman MW, Lichtman MA. The paradox of the neutrophil's role in tissue injury. J. Leukoc. Biol. 2011; 89: 359-372.

10. Putri TL, Ganie RA, Rambe AS. Neutrophil-lymphocyte ratio and high sensitivity c-reactive protein as ischemic stroke outcome predictor. Indonesian Journal of Clinical Pathology and Medical Laboratory, 2017; 23(3): 240-245.

11. Ishikawa $\mathrm{N}$, Kobayashi $\mathrm{Y}$, Fujii $\mathrm{Y}$, Kobayashi $\mathrm{M}$. Increased interleukin- 6 and high-sensitivity C-reactive protein levels in pediatric epilepsy patients with frequent, refractory generalized motor seizures. Seizure, 2015; 25: 136-140.

12. Powers WJ, Rabinstein AA, Ackerson T, Adeoye OM, Bambakidis NC, Becker K, et al. Guidelines for the early management of patients with acute ischemic stroke. A guideline for healthcare professionals from the American Heart Association/American Stroke Association. Stroke, 2018; 49: e63-e71.

13. Guo J, Guo J, Li J, Zhou M, Qin F, Zhang S, et al. Statin treatment reduces the risk of post-stroke seizures. Neurology, 2015; 85: 1-7.

14. Zhao J, Zhang X, Dong L, Wen Y, Cui L. The many roles of statin in ischemic stroke. Current Neuropharmacology, 2014; 12: 564-574.

15. Gunadharma S, Kusumastuti K, Bintoro AC. EEG pada epilepsi. Dalam: Kumastuti K, Gunadharma S, Kustiowati E. (Ed.). Pedoman tatalaksana epilepsi. Surabaya, Airlangga University Press, 2014; 85-91.

16. Hansson, GK. Inflammation, atherosclerosis, and coronary artery disease. N Engl J Med, 2005; 352(16): 1685-95.

17. Ridker PM. C-reactive protein: A simple test to help predict risk of heart attack and stroke. Circulation, 2003; 108: e81-e85.

18. Hillyard DZ, Cameron AJM, McDonald KJ, Thomson J, MacIntyre A, Shiels PG. Simvastatin inhibits lymphocyte function in normal subjects and patients with cardiovascular disease. Atherosclerosis, 2004; 175: 305-313.

19. Kitagawa K, Hosomi N, Nagai Y, Kagimura T, Ohtsuki T, Origasa $\mathrm{H}$. Reduction in high-sensitivity C-reactive protein levels in patients with ischemic stroke by statin treatment: Hs-CRP sub-study in J-STARS. J Atheroscler Thromb, 2017; 24: 1039-1047.

20. Citraro R, Chimirri S, Aiello R, Gallelli L, Trimboli F, Britti $D$, et al. Protective effects of some statins on epileptogenesis and depressive-like behavior in WAG/Rij rats, a genetic animal model of absence epilepsy. Epilepsia, 2014; 55(8): 1284-1291. 2014. 